\title{
An up to 36Gbps Analog Baseband Equalizer and Demodulator for mm-Wave Wireless Communication in $28 \mathrm{~nm}$ CMOS
}

\author{
Oscar Elisio Mattia ${ }^{* \dagger}$, Davide Guermandi ${ }^{\dagger}$, Guy Torfs ${ }^{\dagger \dagger}$, Piet Wambacq ${ }^{* \dagger}$ \\ *ETRO Department \\ Vrije Universiteit Brussel, Pleinlaan 2, Brussel \\ Email: oscar.elisio.mattia@imec.be \\ $\dagger_{\text {imec, Leuven, Belgium }}$ \\ $\ddagger_{\text {imec - Ghent University, Ghent, Belgiun }}$
}

\begin{abstract}
Future mm-Wave wireless links with datarates of 20Gbps and more will result in prohibitive power consumption at the front end of the DSPs. The use of analog or mixed-signal baseband processing, however, can significantly relax the receiver power budget. The most critical block for such a baseband is the decision feedback equalizer, that compensates for the line-of-sight multi-path components and demodulates the signal. In this paper we present, to the authors' best knowledge, the first DFE capable of handling 16QAM data at $9 \mathrm{GHz} \mathrm{RF}$ bandwidth, aggregating all 4 channels of the $60 \mathrm{GHz}$ IEEE802.11ad band and resulting in a maximum datarate of 36Gbps. It is able to compensate for $0.7 x$ cursor amplitude of inter-symbol interference spread over 5 complex taps, while the minimum input $S N R$ is $26 \mathrm{~dB}$. It consumes $138 \mathrm{~mW}$ from a $0.9 \mathrm{~V}$ supply, achieving $3.8 \mathrm{~mW} / \mathrm{Gbps}$ power efficiency including clock distribution.
\end{abstract}

\section{INTRODUCTION}

Future mm-Wave links will require data rates of $20 \mathrm{Gbps}$ and more, over wide bandwidths, unavoidably leading to a large power consumption in the analog baseband section and the digital front-end. Analog demodulation significantly relaxes the requirements for the oversampling ADC and the subsequent digital circuitry. Different baseband functions, such as carrier synchronization, baseband clock recovery, equalization and demodulation, in the analog domain, have been proposed in [1], [2]. The most critical block is the time-domain equalizer, in the form of a decision feedback equalizer (DFE), that compensates for the channel multi-path components (MPC) under practical line-of-sight (LOS) condition. The number of taps depends on the delay spread, which can be as small as $0.5 \mathrm{~ns}$ for very directional and short distance links. In a rough approximation, at least one tap is needed for every symbol period of delay spread. In the case of a $9 \mathrm{GS} / \mathrm{s}$ baseband symbol rate, it means 1 tap for every 110ps of delay spread.

In this paper we present the first DFE capable of equalizing 5 complex taps of inter-symbol interference (ISI) on QPSK/16QAM data, at a maximum data rate of $18 / 36 \mathrm{Gbps}$ respectively, corresponding to an RF bandwidth of $9 \mathrm{GHz}$ that aggregates all 4 channels of the IEEE802.11ad standard. This is realized with I and Q signal paths that can each handle 4PAM signals, leading to 16 possible constellation points to demodulate. To come to a full RX, the circuit is to be preceded by a mm-Wave LNA and IQ direct downconverter, as well as carrier synchronization and baseband clock recovery [2, 4].
These blocks are less critical to meet the bandwidths and data rates mentioned above.

\section{ARCHITECTURE AND CiRCUIT SCHEMATICS}

Before introducing the chosen architecture, it is important to emphasize some of the challenges in designing a DFE for a complex and multi-level modulation scheme, with respect to their more traditional real and binary counterparts found in wireline systems. First and perhaps more obvious are the noise and linearity requirements for the analog part, as well as $3 \mathrm{x}$ more logic gates per coefficient. Second, when looking at the closure of the most critical first tap traditional techniques used to relax the timing budget, such as time interleaving or half-rate sampling require further doubling the number of comparators and digital logic. Third, the coefficient design is also simpler in wireline, where the first coefficient has always positive sign due to the RC nature of the channel. This way an extra XOR gate in series with the comparator is not needed, making the first tap feedback loop shorter and often combined into the comparator structure itself [3]. Finally, cascode isolation [2] that could allow a bigger number of taps is not available because of the $0.9 \mathrm{~V}$ supply of the $28 \mathrm{~nm}$ CMOS process. All of these factors result in greater power consumption, making it unrealistic to achieve the same efficiencies of $1 \mathrm{~mW} / \mathrm{Gbps}$ that state of the art simple wireline equalizers do. Solutions with more advanced equalization, including ADC/DSP-based designs, are closer to and often above a budget of $10 \mathrm{~mW} / \mathrm{Gbps}$.

\section{A. 16QAM 5-tap DFE Core}

Given the reasons mentioned above, the DFE is implemented as a direct full-rate architecture having 5 complex taps current summed in a resistive load, as shown in Figure 1 (Ipath only).

The incoming baseband signal $V_{I N}$ containing all the ISI is converted into current $I_{M A I N}$ by the differential pair. Three comparators, each with a different offset, are placed at the summing node, detecting the four levels of a 4PAM modulation. The nominal ISI-free input amplitude is $200 \mathrm{mVpp}$ for the \pm 3 levels. The output of the comparators feed a delay line, that is tapped by the DFE coefficients. The total tap current $I_{T A P S}$ is subtracted from $I_{M A I N}$ to generate an ISI free eye-diagram. Note that, as an IQ receiver, the in-phase 


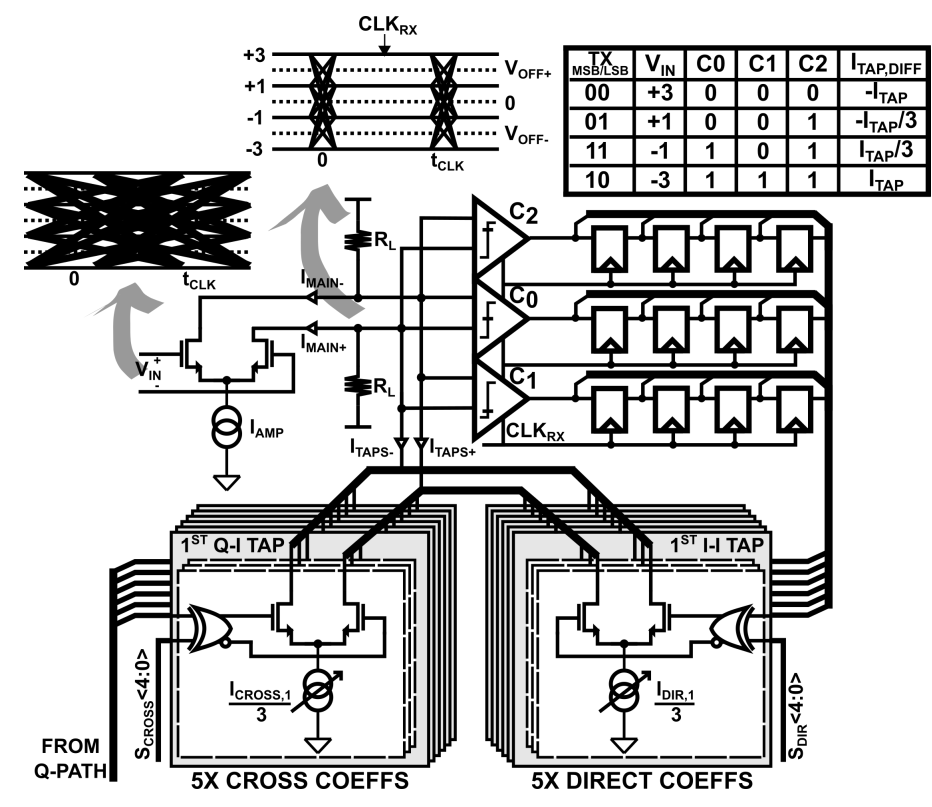

Fig. 1. Schematics of I-path 5-tap complex DFE.

(quadrature) DFE also contain cross coefficients, that come from the Q(I)-path delay line, and are used to compensate for the complex characteristic of the MPCs. Every coefficient is made of 3 unit cells, each containing a 7b current-steering DAC driven by the data bits and coefficient sign selection logic. The sum of the currents is either $\pm 3 I_{T A P}$ or $\pm 1 I_{T A P}$, according to the previous incoming bits. All DFE logic is built in CML style for a maximum clock frequency of $9 \mathrm{GHz}$.

\section{B. 1st Tap Timing Closure}

To ensure timing closure of the first tap of the DFE, the settling time of the analog node and the digital propagation time has to be smaller than a clock period, 110ps. Assuming that the analog settling takes $75 \%$ of that time (more than $30 \mathrm{GHz}$ of bandwidth for $3 \tau$ settling), there is only $27 \mathrm{ps}$ left for the digital gates, including the delays of both comparator and XOR sign-select gate, as well as the comparator setup time. It would be the equivalent of a flash ADC comparator with a sampling clock of more than $40 \mathrm{GHz}$, and therefore clearly needs some attention.

Figure 2 shows the comparator schematics, composed of two static CML latches in master-slave configuration. Fighting offset across PVT with sizing is very inefficient for these highspeed applications. The traditional approach is to use a currentDAC at the output of the first latch to calibrate this offset after production. The problem with this compensation scheme is that it unbalances the first latch output, leading to asymmetric rise/fall times, depending on the sign of the input signal. This will not only make the comparator slower for the unfavored transition, but also affect the settling of the tap currents. To mitigate this imbalance effect we propose the use of different threshold devices on the sensing pair of comparators $C_{1}$ and $C_{2}$ to embed an initial offset $V_{O F F}$, bringing it on average closer to the desired level of \pm 2 (despite PVT variations), considerably speeding up the transition of the comparator with respect to the current-DAC only topology.
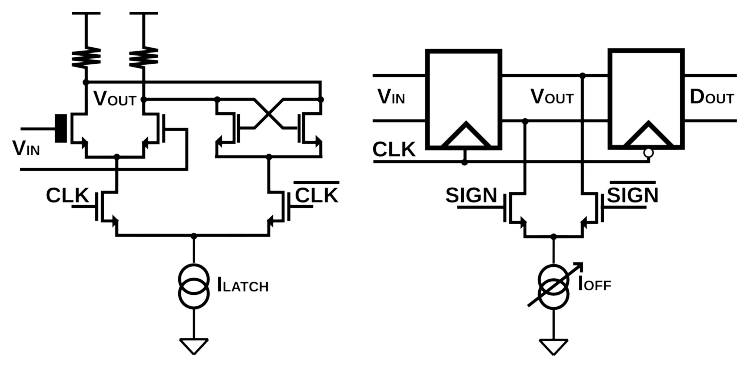

Fig. 2. CML Latch and Comparator schematics, including different $V_{T H}$ devices and offset compensating current-DAC.

Shown in Figure 3 is a post-layout extraction simulation result at the input of the DFE and at the summing node in front of the comparators, while canceling $0.3 \mathrm{x}$ of cursor ISI on the 1 st direct coefficient of the DFE, clocked at 9GHz. While on the amplifier input no distinguishable 4PAM signal is visible, at the comparators' input the tap currents have already subtracted the ISI, leading to a clear open eye. The extra levels around the nominal $\pm 1 / 3$ are the result of a first direct coefficient greater than the applied ISI, which compensates for the approximately $10 \mathrm{mV}$ hysteresis found on this type of static latches.
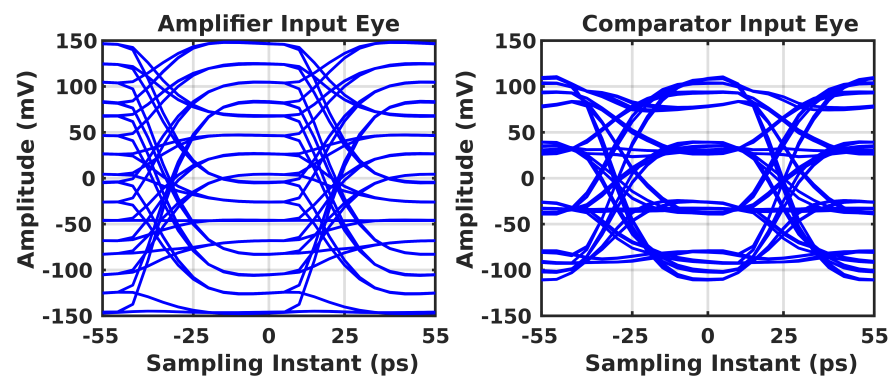

Fig. 3. DFE summing amplifier input and output nodes, showing the effect of equalization and hysteresis compensation for the first direct tap of the DFE.

\section{Chip Architecture and Building Blocks}

The chip is designed for three test modes (Figure 4): (1) is for external testing with an Arbitrary Waveform Generator (AWG) and a Logic State Analyzer (LSA). It has as inputs the I and Q downconverted signals, which are terminated on chip at $50 \Omega$ and $\mathrm{AC}$ coupled, followed by an input buffer. The output bits of the comparators are decoded into MSB/LSB, demuxed by 4, converted from CML (white) to CMOS (grey) and buffered off-chip; test mode (2) is fully integrated, needing only TX and RX clocks, containing two 4PAM PRBS9 generators and a BER tester (see Figure 5). Finally, test mode (3) outputs the PRBS9 generators signal for verification purposes.

The 4PAM PRBS9 generator produces uncorrelated MSB and LSB bits, which are Gray coded and feed a transmitter cell with two 7b DACs. To compose the 16QAM signal they are randomly time-shifted between I and Q paths, resulting again in uncorrelated sequences from the point of view of the RX. On the other side, the BER tester receives the DFE MSB/LSB I/Q signals, that are fed into the same PRBS generator of the TX side, operating in open loop. Their output is masked by a clock and divided by counters, converted to CMOS and buffered off-chip. This way, a transition of the output represents a programmable number of errors of the DFE. 


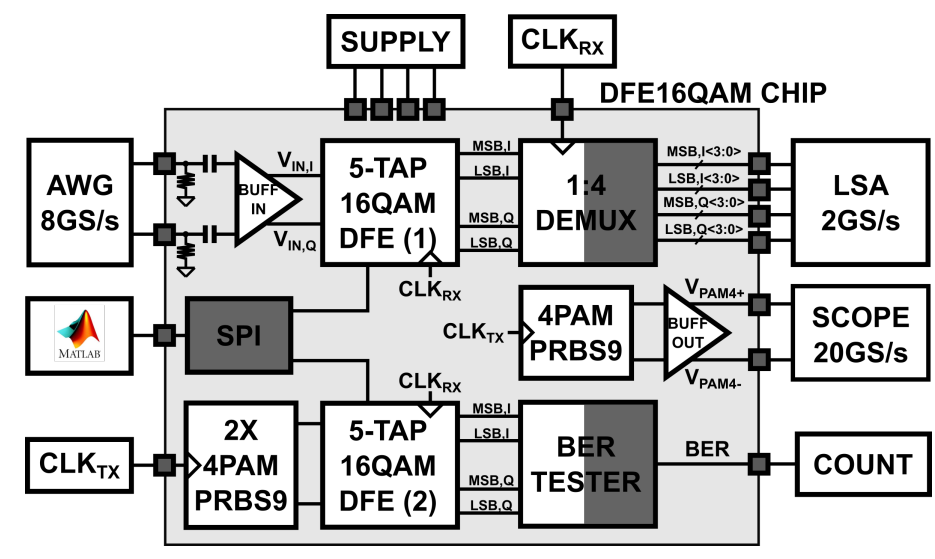

Fig. 4. DFE16QAM chip. Test modes (1) DFE AWG signal and LSA; (2) Integrated PRBS generator and BER tester; (3) Output of PRBS generator.

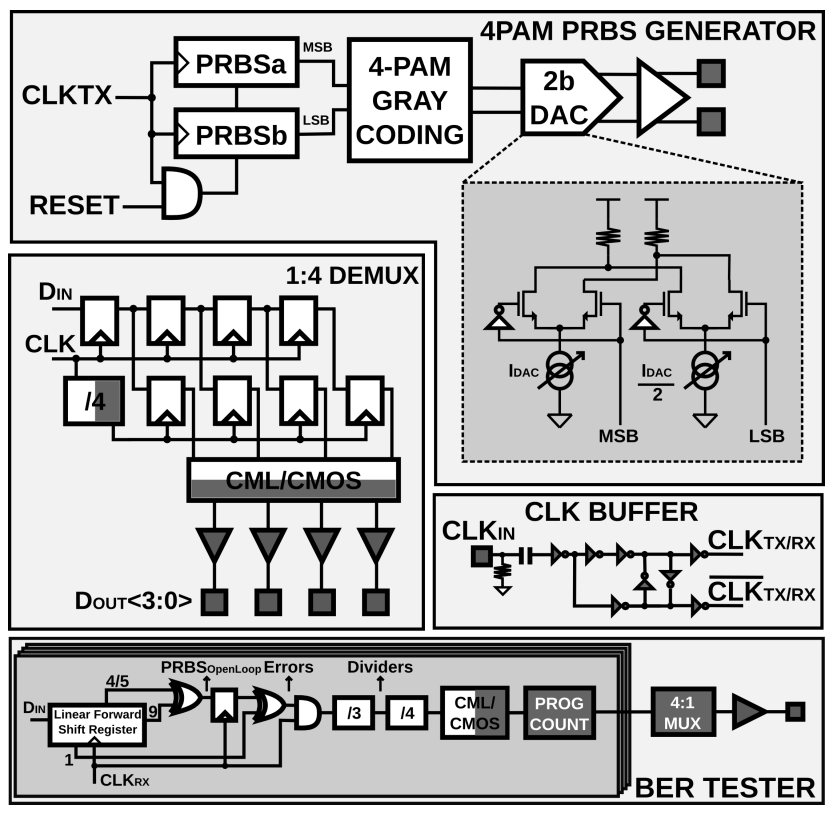

Fig. 5. Auxiliary blocks: 4PAM PRBS9 generator, 1:4 output demux, CLK buffers, integrated BER tester.

\section{MeAsurement Results}

Figure 6 shows the die micrograph, fabricated in $28 \mathrm{~nm}$ CMOS technology. The 16QAM 5-tap complex DFE occupies a total active area of $410 \times 450 \mu \mathrm{m}^{2}$.

Figure 7 shows experimental BER results vs. sampling instant for the integrated test mode (2) of Figure 4, before and after offset calibration, as well as hysteresis compensation (where the first direct coefficient is made to compensate for roughly $10 \mathrm{mV}$ of hysteresis). The receiver makes no mistakes for 1E9 bits on $0.86 / 0.32$ unit interval (UI) at $18 / 36 \mathrm{Gbps}$, for QPSK/16QAM modulations, respectively, successfully validating the most critical 1 st tap closure and all of the proposed test structures. The fact that the receiver needs hysteresis compensation for 16QAM mode can indicate the presence of residual ISI due to finite bandwidth of the PRBS9 generators, or a higher hysteresis value than predicted by simulation.

To include emulated SNR and ISI conditions, the

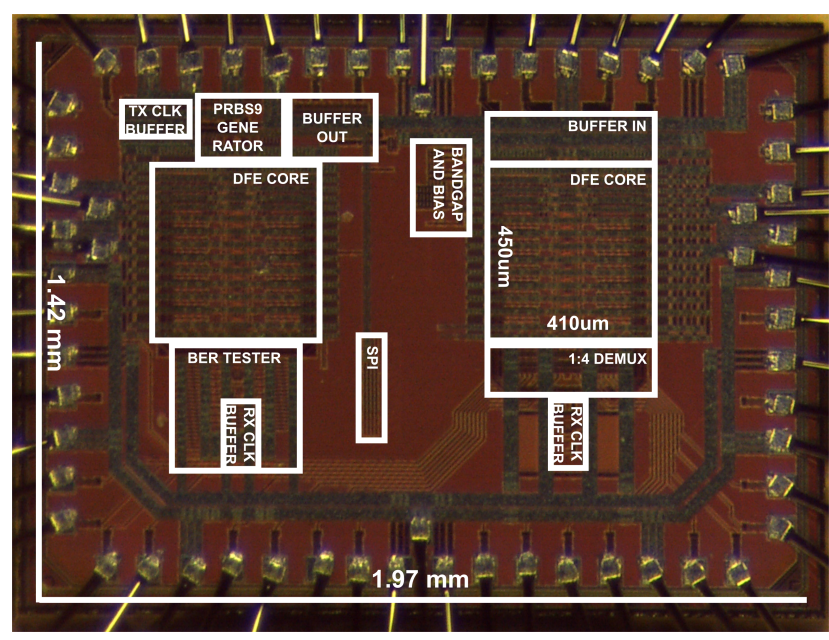

Fig. 6. Die photo in 28nm CMOS. 16QAM 5-tap DFE area is $410 \times 450 \mu \mathrm{m}^{2}$

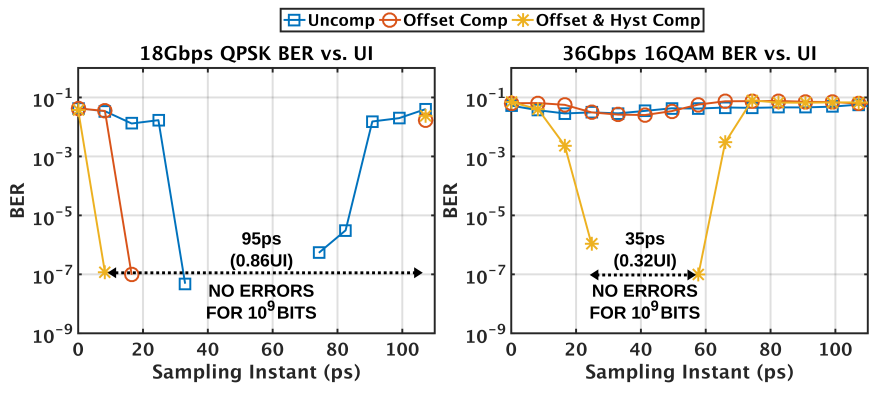

Fig. 7. BER vs. Sampling Instant curves for $18 \mathrm{Gbps}$ QPSK and $36 \mathrm{Gbps}$ 16QAM data, including comparators' offset and hysteresis calibration.

AWG+LSA setup (1) of Figure 4 is needed. In this scenario we are constrained to $16 \mathrm{Gbps}$ (due to measurement setup limitations) for both modulation schemes. As shown in Figure 8 (top), the proposed DFE is able to compensate for $0.7 \mathrm{x}$ cursor amplitude of ISI while making no errors for 1E6 bits, with 0.6/0.36UI aperture, for QPSK/16QAM respectively. The noise performance (without any added ISI) is shown on Figure 8 (bottom), needing a minimum SNR of $15 / 26 \mathrm{~dB}$ for QPSK/16QAM data, respectively.

The channel impulse response and resulting constellation diagrams, as loaded into the AWG memory, are shown in Figure 9. The dotted lines represent the decision thresholds for an ideal detector. As can be seen, $0.7 x$ cursor ISI is not enough to produce errors for a QPSK signal, while it is impossible to demodulate the 16QAM signal under the same conditions. We therefore propose a different metric, that ratios the total ISI with respect to the constellation decision margin (1 for QPSK and 0.33 for 16QAM). An ideal detector would, therefore, be able to demodulate any signal with a relative ISI $<1$. For the case of Figure 9 it results in a relative ISI of $0.7 \mathrm{x} / 2.3 \mathrm{x}$ for the QPSK/16QAM case, respectively, and makes more sense for comparison purposes.

A summary of the DFE core power consumption is shown in Table I. As stated previously, the digital gates and clock buffering power consumption increases significantly when going from a binary modulation to a multi-level one. Still, the circuit is able to achieve reasonable power efficiency of 

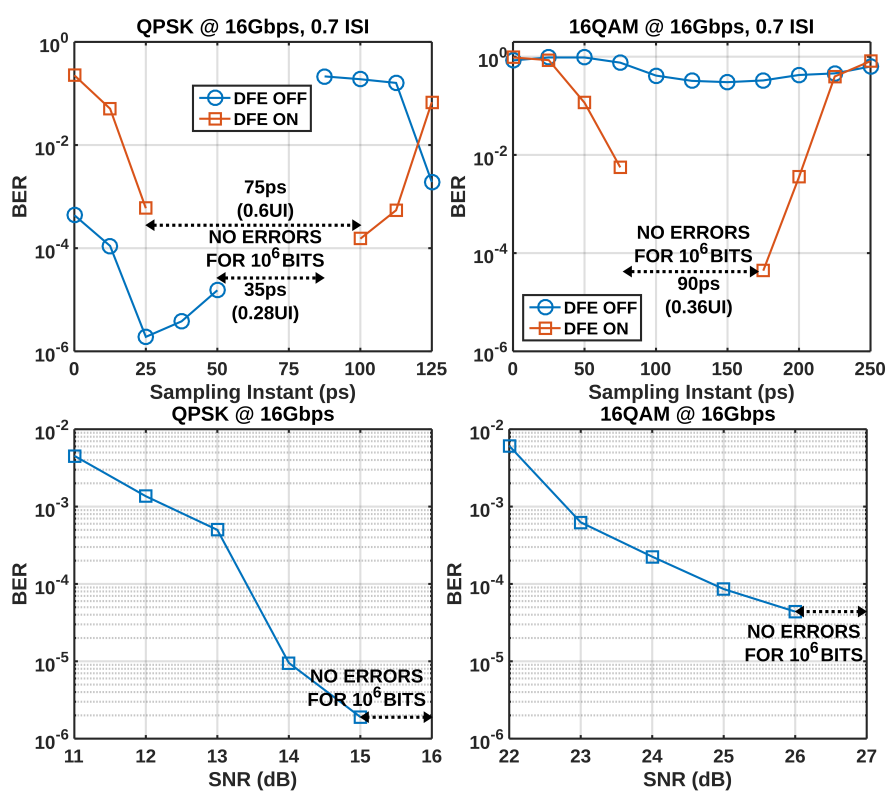

Fig. 8. Measured BER vs. sampling instant, with 0.7ISI (top) and SNR with no ISI (bottom). QPSK/16QAM data, both at $16 \mathrm{Gbps}$ (8GS/s and 4GS/s, left and right, respectively).
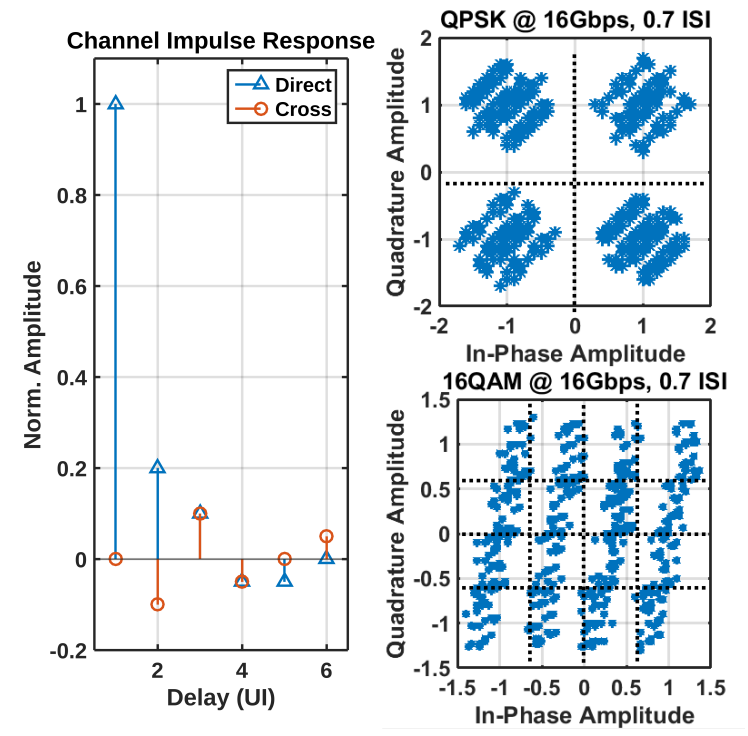

Fig. 9. Channel impulse response containing $0.7 \mathrm{x}$ cursor ISI and resulting constellations for QPSK and 16QAM data.

$3.8 \mathrm{~mW} / \mathrm{Gbps}$ for the 16QAM case. A comparison against the wireless state of the art is presented in Table II. With respect to previous implementations, we have doubled the clock frequency and modulation complexity, resulting in a total datarate that is almost $4 \mathrm{x}$ higher. The penalty on the ISI cancellation is mainly due to a smaller number of taps, since relative to the constellation margin, it is essentially the same. Maintaining noise and linearity requirements while operating with a $0.9 \mathrm{~V}$ supply is challenging, and obviously impacts the overall power consumption. Still, the more advanced and faster node of $28 \mathrm{~nm}$ certainly helps, and a complete receiver baseband, including variable gain amplifier, carrier and baseband clock recovery, should consume no more than $10 \mathrm{~mW} / \mathrm{Gbps}$.
TABLE I. POWER CONSUMPTION BREAKDOWN.

\begin{tabular}{c|c|c|c|c|c|c|c}
\hline \multicolumn{2}{c|}{ Spec / Block } & DFE Amp & Comparator & FF & XOR & CLK Buffer & Unit \\
\hline \hline \multicolumn{2}{c|}{ Avg Power } & 11.7 & 4.5 & 1.44 & 0.72 & 36 & $\mathrm{~mW}$ \\
\hline \multirow{2}{*}{ Multiplier } & QPSK & $2 \mathrm{x}$ & $2 \mathrm{x}$ & $8 \mathrm{x}$ & $8 \mathrm{x}$ & $0.33 \mathrm{x}$ & - \\
\cline { 2 - 8 } & $16 \mathrm{QAM}$ & $2 \mathrm{x}$ & $6 \mathrm{x}$ & $24 \mathrm{x}$ & $24 \mathrm{x}$ & $1 \mathrm{x}$ & - \\
\hline \multirow{2}{*}{ Total Power } & QPSK & 23.4 & 9 & 11.5 & 5.8 & 11.9 & $62 \mathrm{~mW}$ \\
\cline { 2 - 8 } & $16 \mathrm{QAM}$ & 23.4 & 27 & 34.6 & 17.3 & 36 & $138 \mathrm{~mW}$ \\
\hline
\end{tabular}

TABLE II. WIRELESS RX STATE OF THE ART COMPARISON.

\begin{tabular}{c|c|c|c|c}
\hline Specification & {$[4]$} & {$[1]$} & {$[2]$} & Proposed \\
\hline \hline Technology & $90 \mathrm{~nm} 1.2 \mathrm{~V}$ & $65 \mathrm{~nm} 1.2 \mathrm{~V}$ & $65 \mathrm{~nm} \mathrm{LP} 1.2 \mathrm{~V}$ & $\mathbf{2 8 n m ~ 0 . 9 V}$ \\
\hline Symbol Rate & $5 \mathrm{GS} / \mathrm{s}$ & $5 \mathrm{GS} / \mathrm{s}$ & $4 \mathrm{GS} / \mathrm{s}$ & $\mathbf{9 ~ G S / s}$ \\
\hline Modulation & QPSK & QPSK & QPSK & $\mathbf{1 6 Q A M}$ \\
\hline Data Rate & $10 \mathrm{Gbps}$ & $10 \mathrm{Gbps}$ & $8 \mathrm{Gbps}$ & $\mathbf{3 6}$ Gbps \\
\hline $\begin{array}{c}\text { DFE } \\
\text { Architecture }\end{array}$ & $\begin{array}{c}\text { Unrolled } \\
\text { half-rate }\end{array}$ & $\begin{array}{c}\text { Unrolled } \\
\text { half-rate }\end{array}$ & $\begin{array}{c}\text { Unrolled } \\
\text { half-rate }\end{array}$ & $\begin{array}{c}\text { Direct } \\
\text { full-rate }\end{array}$ \\
\hline Number of Coeffs & 10 & 40 & 100 & $\mathbf{1 0}$ \\
\hline Coeff Resolution & 6 bits & 7 bits & 8 bits & $\mathbf{7}$ bits \\
\hline ISI (wrt cursor) & - & $2.5 \mathrm{X}$ & $2 \mathrm{X}$ & $\mathbf{0 . 7 X} *$ \\
\hline ISI (relative) & - & $2.5 \mathrm{X}$ & $2 \mathrm{X}$ & $\mathbf{2 . 3 X}$ \\
\hline Power (mW) & 12 & 14 & 42 & $\mathbf{1 3 8}$ \\
\hline Efficiency (mW/Gbps) & 1.2 & 1.4 & 5.25 & $\mathbf{3 . 8}$ \\
\hline
\end{tabular}

${ }^{1}$ Includes 32 FFE coefficients; ${ }^{2}$ Includes phase rotator; *Measured at $16 \mathrm{Gbps}$.

\section{CONCLUSion}

The chip demonstrates, for the first time, analog demodulation and equalization of 16QAM signals at a total data rate up to $36 \mathrm{Gbps}$, doubling both the clock frequency and the modulation complexity when compared to previous implementations, while maintaining comparable power efficiency of $3.8 \mathrm{~mW} / \mathrm{Gbps}$. It implements a complex 5-tap DFE with resistive summing and full-rate architecture, as well as 1:4 demuxing and integrated test structures. It is a first step towards a complete baseband for future power-efficient $\mathrm{mm}$ Wave receivers.

\section{ACKNOWLEDGMENT}

The authors would like to thank Luc Pauwels and Hans Suys for lab support.

\section{REFERENCES}

[1] C. Thakkar, L. Kong, K. Jung, A. Frappe, and E. Alon, "A $10 \mathrm{~Gb} / \mathrm{s}$ $45 \mathrm{~mW}$ adaptive $60 \mathrm{GHz}$ baseband in $65 \mathrm{~nm}$ CMOS," IEEE Journal of Solid-State Circuits, vol. 47, no. 4, pp. 952-968, 2012.

[2] C. Thakkar, N. Narevsky, C. D. Hull, and E. Alon, "Design Techniques for a Mixed-Signal I/Q 32-Coefficient Rx-Feedforward Equalizer, 100Coefficient Decision Feedback Equalizer in an $8 \mathrm{~Gb} / \mathrm{s} 60 \mathrm{GHz} 65 \mathrm{~nm} \mathrm{LP}$ CMOS Receiver," IEEE Journal of Solid-State Circuits, vol. 49, no. 11, pp. 2588-2607, 2014.

[3] J. Lee, P.-c. Chiang, P.-j. Peng, L.-Y. Chen, and C.-C. Weng, "Design of $56 \mathrm{~Gb} / \mathrm{s}$ NRZ and PAM4 SerDes Transceivers in CMOS Technologies," IEEE Journal of Solid-State Circuits, vol. 50, no. 9, pp. 2061-2073, Sep. 2015.

[4] C. Marcu, D. Chowdhury, C. Thakkar, J. D. Park, L. K. Kong, M. Tabesh, Y. Wang, B. Afshar, A. Gupta, A. Arbabian, S. Gambini, R. Zamani, E. Alon, and A. M. Niknejad, "A $90 \mathrm{~nm}$ CMOS low-power $60 \mathrm{GHz}$ transceiver with integrated baseband circuitry," IEEE Journal of SolidState Circuits, vol. 44, no. 12, pp. 3434-3447, 2009. 